\title{
On the determining number and the metric dimension of graphs
}

\author{
José Cáceres * \\ Department of Statistics and Applied Mathematics \\ University of Almería, Almería, Spain \\ jcaceres@ual.es \\ Delia Garijo * \\ Department of Applied Mathematics I \\ University of Seville, Seville, Spain \\ dgarijo@us.es \\ María Luz Puertas * \\ Department of Statistics and Applied Mathematics \\ University of Almería, Almería, Spain \\ mpuertas@ual.es \\ Carlos Seara $^{\dagger}$ \\ Department of Applied Mathematics II \\ Universitat Politècnica de Catalunya, Barcelona, Spain \\ carlos.seara@upc.edu
}

Submitted: Oct 14, 2008; Accepted: Apr 5, 2010; Published: Apr 19, 2010

Mathematics Subject Classification: 05C25, 05C85

\begin{abstract}
This paper initiates a study on the problem of computing the difference between the metric dimension and the determining number of graphs. We provide new proofs and results on the determining number of trees and Cartesian products of graphs, and establish some lower bounds on the difference between the two parameters.
\end{abstract}

\footnotetext{
*Research supported by projects MEC MTM2008-05866-C03-01 and PAI P06-FQM-01649.

${ }^{\dagger}$ Research supported by projects MEC MTM2006-01267 and DURSI 2005SGR00692.
} 


\section{Introduction}

Let $G$ be a connected graph ${ }^{1}$. A set of vertices $S$ is a determining set of a graph $G$ if every automorphism of $G$ is uniquely determined by its action on $S$. The determining number is the smallest size of a determining set.

Determining sets of connected graphs were introduced by Boutin [4], where ways of finding and verifying determining sets are described. The author also gives natural lower bounds on the determining number of some graphs, developing a complete study on Kneser graphs. Concretely, tight bounds for their determining numbers are obtained and all Kneser graphs with determining number 2, 3 or 4 are provided. Recently, Boutin [5] has studied the determining number of Cartesian products of graphs, paying special attention to powers of prime connected graphs. Moreover, she computes the determining number of the hypercube $Q_{n}$.

Independently, Harary [13] and Erwin and Harary [11] defined an equivalent set and an equivalent number that they called the fixing set and the fixing number, respectively. They found necessary and sufficient conditions for a tree to have fixing number 1 , showing that for every tree there is a minimum fixing set consisting only of leaves of the tree. This approach has its roots on the notion of symmetry breaking which was formalized by Albertson and Collins [2] and Harary [13]. In that approach, a subset of vertices is colored in such a way that the automorphism group of the graph is "destroyed", i.e., the automorphism group of the resulting structure is trivial.

For recent papers on determining sets see the works by Albertson, Boutin, Collins, Erwin, Gibbons, Harary, and Laison $[1,4,5,9,11,12,13]$. Determining sets are frequently used to identify the automorphism group of a graph. Furthermore, they are obtained by using its connection with another well-known parameter of graphs: the metric dimension or location number.

A set of vertices $S \subseteq V(G)$ resolves a graph $G$, and $S$ is a resolving set of $G$, if every vertex is uniquely determined by its vector of distances to the vertices of $S$. A resolving set $S$ of minimum cardinality is a metric basis, and $|S|$ is the metric dimension of $G$.

Resolving sets in graphs were first independently defined by Slater [25], and Harary and Melter [14]. They have since been widely studied, arising in several areas including coin weighing problems, network discovery and verification, robot navigation, connected joins in graphs, and strategies for Mastermind game. The works developed by Cáceres et al. [6] and Hernando et al. [15] provide recent results and an extensive bibliography on this topic.

Besides the above-mentioned papers, Khuller et al. provide in [19] a formula and a linear time algorithm for computing the metric dimension of a tree. They also obtain an upper bound for the metric dimension of the $d$-dimensional grid, showing that to compute the metric dimension of an arbitrary graph is an NP-hard problem. On the other hand, Chartrand et al. [8] characterize the graphs with metric dimension $1, n-1$, and $n-2$.

\footnotetext{
${ }^{1}$ Graphs in this paper are finite, undirected and simple. The vertex-set and edge-set of a graph $G$ are denoted by $V(G)$ and $E(G)$, respectively. The order of $G$ is the number of its vertices, written as $|V(G)|$. The distance between vertices $v, w \in V(G)$ is denoted by $d(v, w)$. For more terminology we follow [26].
} 
They also provide a new proof for the metric dimension of trees and unicyclic graphs. See also [22] for tight bounds on the metric dimension of unicyclic graphs.

Some other important works related to the metric dimension have to do with wheels and Cartesian products. Shanmukha and Sooryanarayana [24] compute this parameter for wheels, and for graphs constructed by joining, in a certain way, wheels with paths, complete graphs, etc. The metric dimension of Cartesian products of graphs has been studied independently by Peters-Fransen and Oellermann [21] and by Cáceres et al. [6].

Taking into account that the determining number is always less or equal than the metric dimension, we come now to our main question: Can the difference between both parameters of a graph of order $n$ be arbitrarily large? This question turns out to be interesting since an automorphism preserves distances and resolving sets are determining sets (see for instance [4, 11]). It arises first as an open problem in [4], and its answer has led us to a number of results on the determining number of some families of graphs in which the metric dimension is known.

A brief plan of the paper is the following. Section 2 recalls some definitions and basic tools. In Section 3, we study the determining number of trees, providing a linear time algorithm for computing minimum determining sets. We also show that there exist trees for which the difference between the determining number and the metric dimension is arbitrarily large. Section 4 focuses on computing the determining number of Cartesian products of graphs, also evaluating the difference between the two parameters. Finally, in Section 5, we provide the family of graphs which attains, until now, the best lower bound on the difference between the metric dimension and the determining number.

\section{Definitions and tools}

An automorphism of $G, f: V(G) \rightarrow V(G)$, is a bijective mapping such that

$$
f(u) f(v) \in E(G) \Longleftrightarrow u v \in E(G) .
$$

As usual Aut $(G)$ denotes the automorphism group of $G$. Every automorphism is also an isometry, i.e., it preserves distances.

The ideas of determining set and resolving set have already been introduced in the previous section. The following are the precise and more technical definitions provided in [4] and [14, 25] (see also [8, 19, 22]).

Definition 1. [4] A subset $S \subseteq V(G)$ is said to be a determining set of $G$ if whenever $g, h \in \operatorname{Aut}(G)$ so that $g(s)=h(s)$ for all $s \in S$, then $g(v)=h(v)$ for all $v \in V(G)$. The smallest size of a determining set of $G$, denoted by $\operatorname{Det}(G)$, is called the determining number of $G$.

An equivalent definition of determining set is provided by Boutin in [4] by using the concept of pointwise stabilizer of $S$ as follows. For any $S \subseteq V(G)$,

$$
\operatorname{Stab}(S)=\{g \in \operatorname{Aut}(G) \mid g(v)=v, \forall v \in S\}=\bigcap_{v \in S} \operatorname{Stab}(v) .
$$


Proposition 1. [4] $S \subseteq V(G)$ is a determining set of $G$ if and only if $\operatorname{Stab}(S)=\{i d\}$.

Some examples of graphs whose determining number can be easily computed are the following. An extreme is a minimum determining set of a path $P_{n}$ and $\operatorname{so} \operatorname{Det}\left(P_{n}\right)=1$. Any pair of non-antipodal vertices is a determining set of a cycle, thus $\operatorname{Det}\left(C_{n}\right)=2$. A minimum determining set of the complete graph $K_{n}$ is any set containing all but one vertex, and hence $\operatorname{Det}\left(K_{n}\right)=n-1$. The determining number of the multipartite complete graph $K_{n_{1}, n_{2}, \ldots, n_{s}}$ can be also easily computed, since a minimum determining set contains $n_{j}-1$ vertices of each of the $s$ classes,

$$
\operatorname{Det}\left(K_{n_{1}, n_{2}, \ldots, n_{s}}\right)=\left(n_{1}+n_{2}+\cdots+n_{s}\right)-s
$$

Observe that every graph has a determining set. It suffices to consider any set containing all but one vertex. Thus, $\operatorname{Det}(G) \leqslant|V(G)|-1$. The only connected graphs with $\operatorname{Det}(G)=|V(G)|-1$ are the complete graphs. On the other hand, a graph $G$ has $\operatorname{Det}(G)=0$ if and only if $G$ is an identity graph, i.e., $\operatorname{Aut}(G)$ is trivial. Those graphs are also called asymmetric graphs (Albertson and Collins [2] use the term rigid graphs. In fact, almost all graphs are rigid [3], hence most graphs have determining number 0).

The characterization of those graphs with $\operatorname{Det}(G)=1$ is observed by Erwin and Harary [11] as follows: Let $G$ be a nonidentity graph. Then $\operatorname{Det}(G)=1$ if and only if $G$ has an orbit of cardinality $|\operatorname{Aut}(G)|$. They also point out that the group of automorphisms of a graph with $\operatorname{Det}(G)=1$ can be arbitrarily large: For every positive integer $t$, there is a graph $G_{t}$ with determining number 1 and $\left|\operatorname{Aut}\left(G_{t}\right)\right|=t$.

The metric dimension is formally defined as follows.

Definition 2. [14, 25] A set of vertices $S$ resolves a graph $G$ if every vertex of $G$ is uniquely determined by its vector of distances to the vertices in $S$, that $i s, d(u, s) \neq d(v, s)$ for all $s \in S$ and $u, v \in V(G)$ with $u \neq v$. The metric dimension of $G$, denoted by $\beta(G)$, is the minimum cardinality of a resolving set of $G$.

The following result was independently proved by Harary [13], Erwin and Harary [11] (using fixing sets instead of determining sets), and Boutin [4].

Proposition 2. [4, 11, 13] If $S \subseteq V(G)$ is a resolving set of $G$ then $S$ is a determining set of $G$. In particular, $\operatorname{Det}(G) \leqslant \beta(G)$.

Given a graph $G$ of order $n$, the set of all its vertices but one is both a resolving set and a determining set. Moreover, every graph $G$ has both a minimum resolving set and a minimum determining set. Thus,

$$
0 \leqslant \operatorname{Det}(G) \leqslant \beta(G) \leqslant n-1
$$

There are many examples where both parameters are equal. For any graph $G$ of order $n$, it is clear that $0 \leqslant \beta(G)-\operatorname{Det}(G) \leqslant n-2$, and Boutin [4] poses the following question: Can the difference between the determining number and the metric dimension of a graph of order $n$ be arbitrarily large? In order to answer it, we first compute the determining 
number of some specific families of graphs in which the metric dimension is known. More concretely, the two sections following are devoted to study these two parameters, and the difference between them for trees and for Cartesian products of graphs. Throughout this paper, $\beta(G)-\operatorname{Det}(G)$ will be considered as a function of the order of $G$, denoted by $n=|V(G)|$.

\section{Trees}

In this section, we focus on computing minimum determining sets of trees, and comparing the metric dimension with the determining number. We provide bounds on the difference between the two parameters.

Let $T=(V(T), E(T))$ be a $n$-vertex nonidentity tree, $n \geqslant 2$, $\operatorname{Det}(T) \geqslant 1$. Assume, unless otherwise stated, that $T$ is not the path $P_{n}$. Clearly, $\operatorname{Det}\left(P_{n}\right)=1$ and most of the

results proved in this section are trivial in that case. Any set formed by all but one leaf is a determining set of $T$. In fact, the following result holds.

Lemma 1. [11] There exists a minimum determining set of $T$ formed by leaves.

Theorem 1. The determining number of a tree $T$ with at least two vertices satisfies the following statements:

1. $0 \leqslant \operatorname{Det}(T) \leqslant n-2$ and both bounds are tight.

2. Given $n, k \in \mathbb{N}$ with $0 \leqslant k \leqslant n-2$ and $k \neq n-3$, there exists a tree $T$ of order $n$ such that $\operatorname{Det}(T)=k$.

3. (a) There exists a tree $T$ so that $\operatorname{Det}(T)=n-3$ if and only if $n=4$.

(b) A tree $T$ such that $\operatorname{Det}(T)=0$ can only exist if $n=1$ or $n \geqslant 7$.

Proof. The four statements are proved one by one.

1. Obviously, $\operatorname{Det}(T) \geqslant 0$ by definition. Furthermore, $T$ contains at most $n-1$ leaves which implies, by Lemma 1, that the cardinality of a determining set of $T$ is at most $n-2$. The tightness of the bounds will be proved in the next item.

2. Consider $n, k \in \mathbb{N}$ with $1 \leqslant k \leqslant n-4$. Figure 1 shows a $u-v$ path $P_{n-k-1}$ with a group of leaves $\left\{v_{1}, \ldots, v_{k+1}\right\}$ hanging from $v$. Since $d(u, v)>1$, the set $\left\{v_{1}, \ldots, v_{k}\right\}$ is a minimum determining set of that tree. Hence its determining number is $k$.

The star $K_{1, n-1}$ serves as example for $k=n-2$ (Figure 2(b)).

3. (a) $P_{4}$ is an example of tree with order $n$ and having determining number $n-3$. Suppose now on the contrary that there exists a tree $T$ with $n \neq 4$ vertices so that $\operatorname{Det}(T)=n-3$. Thus, there is a minimum determining set of size $n-3$. Moreover, Lemma 1 implies that this minimum set is formed by leaves. Hence $T$ has at least $n-2$ leaves, which leads to the trees illustrated in Figure 2. 


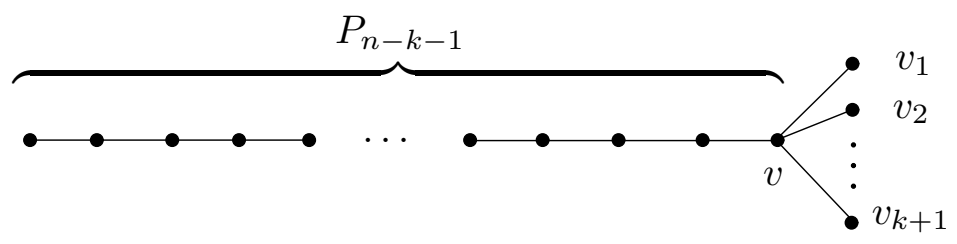

Figure 1: A tree with determining number $k$ formed by adding a group of leaves hanging one endpoint of a path $P_{n-k-1}$.

The contradiction follows from the determining numbers of those graphs. Figure 2(a) shows a tree with determining number $n-4$, and the star (Figure $2(\mathrm{~b}))$ has determining number $n-2$.

(b) For the case $k=0$, it is clear that the automorphism group of the trivial graph is also trivial, thus its determining number is zero. On the other hand, an inspection of the trees with order between 2 and 6 will prove that all of them have some kind of symmetry and thus their automorphism group is not trivial. Finally, when $n \geqslant 7$ we prove that there always exists a tree $T$ with $\operatorname{Det}(T)=0$. That tree $T$ is obtained by identifying one leaf from at least three paths of different lengths. Assume that $f \in \operatorname{Aut}(T)$. Since $T$ contains a unique vertex with degree at least three, it must be fixed by $f$. Moreover, $f$ should map a path onto itself due to the different lengths. Finally, because $f$ is an isometry, it does not change the order of the vertices in a path, thus $f=i d$ and $\operatorname{Det}(T)=0$.

Thus the theorem follows.

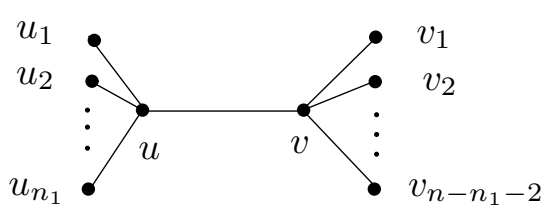

(a)

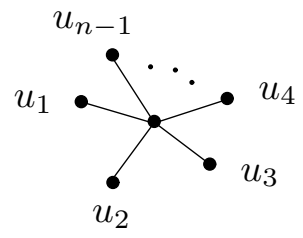

(b)

Figure 2: The two possible $n$-vertex trees with at least $n-2$ leaves.

\subsection{Algorithmic study}

The center of a graph is the subgraph induced by the vertices of minimum eccentricity. The center of a tree is either one vertex $v_{0}$ or one edge $v_{1} v_{2}$ (see $[18,26]$ ). In the first case, $v_{0}$ is the best candidate for being the root of $T$ in order to compute the determining 
number. Indeed, $T$ can be viewed as a rooted tree, i.e., a tree in which one vertex, called the root, is distinguished. In order to study a rooted tree it is natural to arrange the vertices in levels. Thus, the root is at level 0 and its neighbors at level 1 . For each $k>1$, level $k$ contains those vertices adjacent to vertices at level $k-1$, except for those which have already been assigned to level $k-2$. The parent of a vertex at level $i$ for $i>0$, is the vertex adjacent to it at level $i-1$. A child of a vertex $v$ is a vertex of which $v$ is the parent. An ancestor of a vertex $v$ is any vertex that lies on the path from the root to $v$. A descendant of a vertex $v$ is any vertex that lies on the path from $v$ to a leaf.

We design the following algorithm which computes a minimum determining set of a tree $T$ rooted at its center, and $\operatorname{Det}(T)$.

Procedure: Minimum-Determining-Set-Tree

Input: $T=(V(T), E(T))$.

Output: A minimum determining set $S$ for $T$.

1. Preprocess. Apply a linear time algorithm [7] to compute the center of $T$;

If the center of $T$ is the edge $v_{1} v_{2}$ then add a vertex $v_{0}$ adjacent to both $v_{1}$ and $v_{2}$ and delete the edge $v_{1} v_{2}$;

Rename the resulting tree as $T$;

2. Let $T$ be rooted at $v_{0}$, let $n$ be the radius of $T$ and $S:=\emptyset$;

Let all the leaves be labeled with "0";

3. For $i:=n-1$ to 0 do

(a) For each non-leaf vertex $u$ at distance $i$ from $v_{0}$ do

i. If $l$ children of $u$ have the same label, then add $(l-1)$ of them to $S$ and distinguish their labels with subindices;

ii. Label $u$ by concatenating in lexicographic order the labels of its children;

(b) Order lexicographically the labels of the vertices at distance $i$ from $v_{0}$ and relabel them with its position in the order beginning with " 1 ";

The Step 3 of the algorithm is a variation of the linear time algorithm by Hopcroft and Tarjan [16] to check whether two trees are isomorphic. Here, we add the instruction 3(a)i. To clarify how it works, let us take a look at the example in Figure 3.

The tree in Figure 3 has a single-vertex center and radius six. We start by assigning "0" to all the leaves. In the next level (level one), the vertices are labeled as in the original algorithm by Hopcroft and Tarjan. In level two, however, there is a vertex with three children with the same label. In this situation, two of them are added to $S$ (which is represented in the figure as a square surrounding the vertex) and its corresponding labels are distinguished with subindices. The situation is repeated in level three where two vertices have the label (11). In that case, we choose one child in each subtree and mark its labels appropriately. We note that no two ancestors of the vertices labeled $\left(11_{1}\right)$ and $\left(11_{2}\right)$ in level 3 receive the same label. 


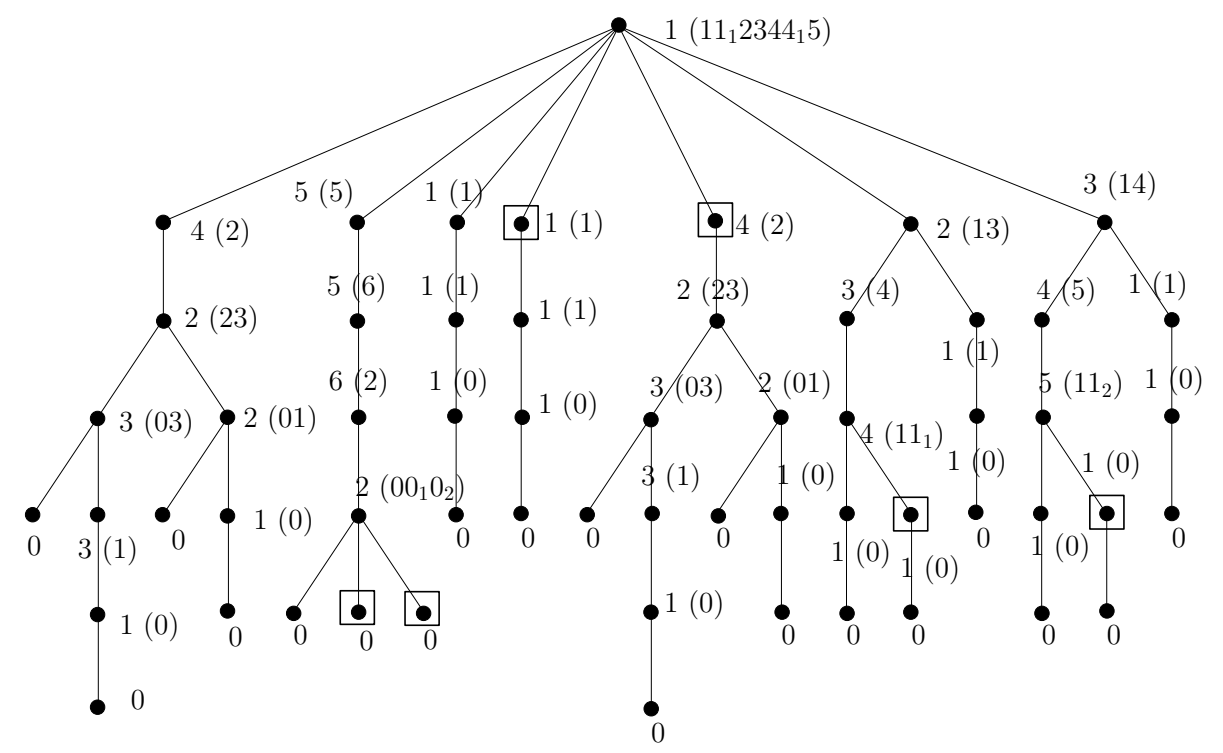

Figure 3: Example of a tree with a single-vertex center and radius six.

Observe that appropriately modified, the algorithm also computes a set $S$ consisting of leaves. Now, we focus our attention to prove that $S$ is effectively a minimum determining set of $T$. In order to do that, we introduce some definitions.

Suppose that $T$ is a tree with a single-vertex centre $v_{0}$. A vertex $x$ of $T$ is the root of a subtree $T_{x}$ of $T$ that consists of all vertices $y$ such that $x$ lies on the $v_{0}-y$ path in $T$. The set $\mathcal{T}$ consists of all subtrees $T_{x}$ of $T$ for which there exists a tree $T_{x^{\prime}}$ isomorphic to $T_{x}$ where $x \neq x^{\prime}$ and such that $x$ and $x^{\prime}$ have the same parent. Let $\mathcal{T}_{0}$ denote the set $T_{x}$ of elements of $\mathcal{T}$ for which $\mathcal{T}$ does not contain a proper subtree of $T_{x}$. So if $T_{1} \in \mathcal{T}$, then either $T_{1} \in \mathcal{T}_{0}$ or it contains an element in $\mathcal{T}_{0}$.

Lemma 2. Given two isomorphic subtrees in $\mathcal{T}_{0}$ with the same parent, at least one of them has a vertex in $S$.

Proof. Let $T_{1}$ and $T_{2}$ be such subtrees and let $u$ be their common parent. During the algorithm's running, at the moment in which the vertex $u$ is explored, its children belonging to $T_{1}$ and $T_{2}$ have the same label since neither $T_{1}$ nor $T_{2}$ contains a subtree in $\mathcal{T}_{0}$ and hence, their labels have not been changed. Therefore, one vertex from at least one of these subtrees is added to $S$, and the result holds true.

Lemma 3. The set $S$ obtained by the algorithm is a determining set of $T$.

Proof. Suppose $f, g \in \operatorname{Aut}(T)$ are such that $f(s)=g(s)$ for all $s \in S$. So $g^{-1} f(s)=s$ for all $s \in S$. We need to show that $g^{-1} f(v)=v$ for all $v \in V(T)$. Since $g^{-1} f$ is an automorphism it follows if $T_{x} \in \mathcal{T}$. Then there is a $T_{y} \in \mathcal{T}$ (possibly $x=y$ ) such that $g^{-1} f\left(T_{x}\right)=T_{y}$ where $x$ and $y$ have the same parent. If $g^{-1} f$ is not the identity, there exist distinct subtrees $T_{x}$ and $T_{y}$ in $\mathcal{T}$ that have a common parent such that $g^{-1} f\left(T_{x}\right)=T_{y}$. 
But, by Lemma 2, either $T_{x}$ or $T_{y}$ contains a vertex of $S$ since they either both belong to $\mathcal{T}_{0}$ or they both contain subtrees that belong to $\mathcal{T}_{0}$. This implies that not all the vertices of $S$ are fixed by $g^{-1} f$, a contradiction.

Lemma 4. The set $S$ constructed by the algorithm is a minimum determining set.

Proof. On the contrary, let $S^{\prime}$ be a determining set of $T$ such that $\left|S^{\prime}\right|<|S|$. Then, by the pigeonhole principle, at least two isomorphic subtrees $T_{1}, T_{2} \in \mathcal{T}_{0}$, with the same root, do not contain a vertex in $S^{\prime}$, and hence there exists an automorphism $f$ different from the identity such that $f\left(T_{1}\right)=T_{2}$. Therefore $S^{\prime}$ is not a determining set.

Remark 1. Once we have obtained the set $S$, it is straightforward how to compute in linear time the corresponding minimum determining set for $T$ only formed by leaves according to Lemma 1. Indeed, if $v \in S$ and if $v$ is not a leaf of $T$, then we replace $v$ by a leaf in the subtree rooted at $v$. Notice that the algorithm above works also for asymmetric trees, i.e., for trees $T$ with $\operatorname{Det}(T)=0$.

As a consequence of the discussion above we have the following result.

Theorem 2. The problem of computing a minimum determining set $S$ of a tree $T$ can be solved in linear time as well as computing a minimum determining set formed by leaves.

Now we turn to the problem of computing the metric dimension of a tree. In Khuller et al. [19], the authors introduce a linear time algorithm for computing the metric dimension of a tree $T$ formed only by leaves. This leads to the natural question: is it always possible to enlarge a a minimum determining set of a tree $T$ formed by leaves in order to obtain a metric basis of $T$ ? Unfortunately, the answer is negative as it is shown in the example of Figure 4.

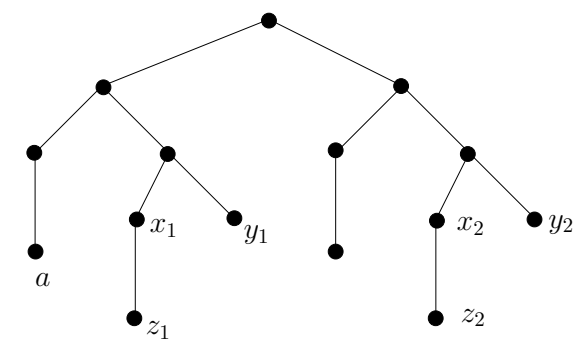

Figure 4: $\{a\}$ is a minimum determining set that cannot be completed to obtain a metric basis.

For the tree in the Figure $4,\{a\}$ is a minimum determining set. Assume that this set can be enlarged with leaves to obtain a metric basis. Since $d\left(a, x_{1}\right)=d\left(a, y_{1}\right)$ and $d\left(a, x_{2}\right)=d\left(a, y_{2}\right)$, this can only be done by choosing either $y_{1}$ or $z_{1}$ and analogously with $y_{2}$ and $z_{2}$. Suppose that we choose $y_{1}$ and $y_{2}$. Then $\left\{a, y_{1}, y_{2}\right\}$ is not a minimum resolving set since $\left\{y_{1}, y_{2}\right\}$ does, and this always occurs for whatever pair of vertices we add. 


\subsection{Lower bounds on $\beta(T)-\operatorname{Det}(T)$}

There exist some examples of trees $T$ for which both parameters $\beta(T)$ and $\operatorname{Det}(T)$ are equal: $\beta\left(P_{n}\right)=\operatorname{Det}\left(P_{n}\right)=1, \beta\left(K_{1, n-1}\right)=\operatorname{Det}\left(K_{1, n-1}\right)=n-2$. The following result shows a construction in which $\beta(T)-\operatorname{Det}(T)$ is $\Omega(\sqrt{n})$. Nevertheless, this bound is a first approximation to the possible value $n-2$.

Proposition 3. There exists a tree $T$ of order $n$ such that $\beta(T)-\operatorname{Det}(T)$ is $\Omega(\sqrt{n})$.

Proof. Consider the tree $T$ formed by connecting a single vertex $u$ to $k$ paths denoted by $P_{m}, P_{m+1}, \ldots, P_{m+k-1}$ with lengths $m, m+1, \ldots, m+k-1$, respectively (see Figure 5 ). Thus $P_{i} \bigcap P_{j}=\{u\}$ for all $i \neq j$.

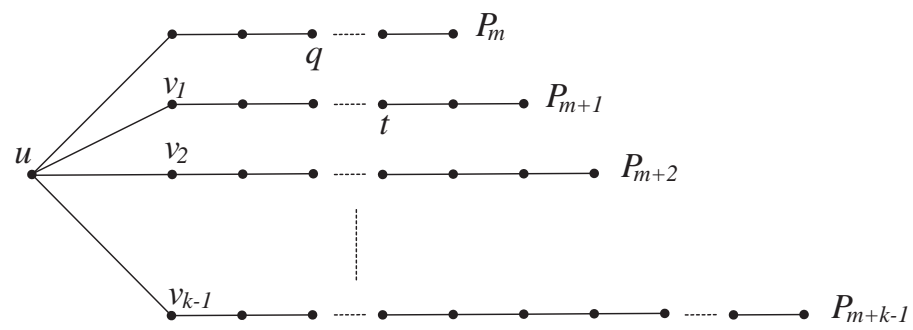

Figure 5: Tree $T$ formed by connecting a single vertex $u$ to $k$ paths of different lengths.

Since all the paths have different length then $\operatorname{Det}(T)=0$. In fact, $\operatorname{Aut}(T)=\{i d\}$, i.e., $T$ is an asymmetric tree. On the other hand, it was shown in $[8,14,19,25]$ that a minimum resolving set for $T$ can be obtained by choosing all but one of the leaves of this tree. Hence, $\beta(T)=k-1$. For $m=1,|V(T)|=n=\frac{k^{2}+k+2}{2}$. Therefore $\beta(T)-\operatorname{Det}(T)$ is $\Omega(\sqrt{n})$.

\section{$4 \quad$ Cartesian products of graphs}

This section arises as a natural consequence of the connection between determining number and metric dimension, and the studies developed by Boutin [5] and Cáceres et al. [6]. Our first purpose is to compute the determining number of some well-known Cartesian products of graphs, for which the metric dimension is known.

The Cartesian product of graphs $G$ and $H$ (see [17]), denoted by $G \square H$, is the graph with vertex set $V(G) \times V(H)$ where $\left(u_{1}, v_{1}\right)$ is adjacent to $\left(u_{2}, v_{2}\right)$ whenever $u_{1}=u_{2}$ and $v_{1} v_{2} \in E(H)$, or $v_{1}=v_{2}$ and $u_{1} u_{2} \in E(G)$. The Cartesian product of automorphisms $f \in \operatorname{Aut}(G)$ and $g \in \operatorname{Aut}(H)$ is the automorphism of $G \square H$ defined as follows: $(f \times$ $g)(u, v)=(f(u), g(v))$ for every $(u, v) \in V(G \square H)$.

Let $S$ be a subset of $V(G \square H)$. The projection of $S$ onto $G$ is the set of vertices $u \in V(G)$ for which there exists a vertex $(u, v) \in S$. The projection of $S$ onto $H$ is defined analogously. A column of $G \square H$ is the set of vertices $\{(u, v) \mid v \in V(H)\}$ for some vertex $u \in V(G)$. A row of $G \square H$ is the set of vertices $\{(u, v) \mid u \in V(G)\}$ for some 
vertex $v \in V(H)$. Thus, each column and each row of $G \square H$ induces a copy of $H$ and $G$, respectively.

Lemma 5. Let $G_{1}, \ldots, G_{p}$ be connected finite graphs and $G=G_{1} \square \cdots \square G_{p}$. For every determining set $S$ of $G$, the projections of $S$ onto $G_{1}, \ldots, G_{p}$ are determining sets of $G_{1}, \ldots, G_{p}$, respectively. In particular,

$$
\operatorname{Det}\left(G_{1} \square \ldots \square G_{p}\right) \geqslant \max \left\{\operatorname{Det}\left(G_{1}\right), \ldots, \operatorname{Det}\left(G_{p}\right)\right\} \text {. }
$$

Proof. Let $S \subseteq V(G)$ be a determining set of $G$. By Proposition 1, we have that $\operatorname{Stab}(S)=\{i d\}$. Consider the projections of $S$ onto $G_{i}$, written as $S_{G_{i}}$, with $1 \leqslant i \leqslant p$. To prove that $S_{G_{i}}$ is a determining set of $G_{i}$ it suffices to show that,

$$
\operatorname{Stab}\left(S_{G_{1}}\right) \times \cdots \times \operatorname{Stab}\left(S_{G_{p}}\right) \subseteq \operatorname{Stab}(S)=\{i d\} .
$$

Given $f_{i} \in \operatorname{Aut}\left(G_{i}\right)$, the Cartesian product $f_{1} \times \cdots \times f_{p} \in \operatorname{Aut}(G)$. Assume that $f_{i}(u)=u$ for all $u \in S_{G_{i}}$. Then $\left(f_{1} \times \cdots \times f_{p}\right)(s)=s$ for all $s \in S_{G_{1}} \times \cdots \times S_{G_{p}}=S$. Hence $f_{1} \times \cdots \times f_{p}=i d$, and we conclude that $\operatorname{Stab}\left(S_{G_{i}}\right)=\{i d\}$ for every $i=1, \ldots, p$.

A straightforward consequence of the above-proved result is that,

$$
\operatorname{Det}\left(G_{1} \square \cdots \square G_{p}\right) \geqslant \max \left\{\operatorname{Det}\left(G_{1}\right), \ldots, \operatorname{Det}\left(G_{p}\right)\right\}
$$

since the determining sets of the projections of $G$ have at most the same order than a minimum determining set of $G$.

We now recall some definitions from Boutin [5] and Sabidusi [23]. The unit graph, denoted by $U$, is the trivial graph given by $V(U)=\{u\}$ and $E(U)=\emptyset$. A graph $G$ is prime with respect to the Cartesian product if it cannot be written as a Cartesian product of two smaller graphs, that is, $G$ is not isomorphic to the unit graph, and if $G \cong Z \square Z^{\prime}$ implies $Z \cong U$ or $Z^{\prime} \cong U$. Two graphs $G$ and $G^{\prime}$ are said to be relatively prime with respect to the Cartesian product if and only if $G \cong H \square Z$ and $G^{\prime} \cong H^{\prime} \square Z$ imply $Z \cong U$, that is, their prime factor decomposition share no common factor. Denote by $G^{m}$ the Cartesian product of $m$ copies of $G$.

The following theorem provides the determining number of the Cartesian product of pairwise relatively prime graphs.

Theorem 3. Let $G_{1}, \ldots, G_{p}$ be connected graphs, and $m_{1}, \ldots, m_{p} \in \mathbb{N}$. If $G_{1}^{m_{1}}, \ldots, G_{p}^{m_{p}}$ are pairwise relatively prime with respect to the Cartesian product, then

$$
\operatorname{Det}\left(G_{1}^{m_{1}} \square \cdots \square G_{p}^{m_{p}}\right)=\max \left\{\operatorname{Det}\left(G_{1}^{m_{1}}\right), \ldots, \operatorname{Det}\left(G_{p}^{m_{p}}\right)\right\} \text {. }
$$

Proof. By Lemma 5, the inequality that remains to prove is that $\operatorname{Det}\left(G_{1}^{m_{1}} \square \cdots \square G_{p}^{m_{p}}\right) \leqslant$ $\max \left\{\operatorname{Det}\left(G_{1}^{m_{1}}\right), \ldots, \operatorname{Det}\left(G_{p}^{m_{p}}\right)\right\}$. To this end, let $S_{1}, \ldots, S_{p}$ be minimum determining sets of $G_{1}^{m_{1}}, \ldots, G_{p}^{m_{p}}$, respectively. Consider a minimum subset of $V\left(G_{1}^{m_{1}} \square \cdots \square G_{p}^{m_{p}}\right)$, denoted by $S$, verifying that each set $S_{i}$ is the projection of $S$ onto $G_{i}^{m_{i}}$. Obviously, $|S|=\max \left\{\left|S_{1}\right|, \ldots,\left|S_{p}\right|\right\}$ and so it suffices to prove that $S$ is a determining set of $G_{1}^{m_{1}} \square \cdots \square G_{p}^{m_{p}}$. 
Let $f, g \in \operatorname{Aut}\left(G_{1}^{m_{1}} \square \cdots \square G_{p}^{m_{p}}\right)$. Since $G_{1}^{m_{1}}, \ldots, G_{p}^{m_{p}}$ are pairwise relatively prime graphs, there exist $f_{i}, g_{i} \in \operatorname{Aut}\left(G_{i}^{m_{i}}\right)$ for $1 \leqslant i \leqslant p$ such that $f=f_{1} \times \cdots \times f_{p}$ and $g=g_{1} \times \cdots \times g_{p}($ see $[23]$ for more details $)$.

Hence,

Assume that $f(s)=g(s)$ for all $s \in S$. Notice that $s=\left(s_{1}, \ldots, s_{p}\right)$ with $s_{i} \in S_{i}$.

$$
f(s)=g(s) \Longleftrightarrow\left(f_{1}\left(s_{1}\right), \ldots, f_{p}\left(s_{p}\right)\right)=\left(g_{1}\left(s_{1}\right), \ldots, g_{p}\left(s_{p}\right)\right) \Longleftrightarrow f_{i}\left(s_{i}\right)=g_{i}\left(s_{i}\right) .
$$

Since the set $S_{i}$ is a minimum determining set of $G_{i}^{m_{i}}$, it follows that $f_{i}\left(v_{i}\right)=g_{i}\left(v_{i}\right)$ for all $v_{i} \in V\left(G_{i}^{m_{i}}\right)$. We conclude that $f(v)=g(v)$ for all $v \in V\left(G_{1}^{m_{1}} \square \cdots \square G_{p}^{m_{p}}\right)$.

It was shown in [23] that every connected finite graph $H$ has an unique prime factor decomposition with respect to the Cartesian product, i.e., $H$ can be written uniquely, up to order, as $H=H_{1}^{m_{1}} \square \cdots \square H_{p}^{m_{p}}$ where the graphs $H_{i}$ are connected, prime, and distinct. Since $H_{1}^{m_{1}} \square \cdots \square H_{p}^{m_{p}}$ is a maximal decomposition of $H$ into relatively prime factors, $\operatorname{Det}(H)=\max \left\{\operatorname{Det}\left(H_{1}^{m_{1}}\right), \ldots, \operatorname{Det}\left(H_{p}^{m_{p}}\right)\right\}$. Thus Theorem 1 of [5], for Cartesian product of prime graphs, can be deduced from Theorem 3, for Cartesian product of pairwise relatively prime graphs. Although both results are similar, the arguments of the proofs are different. To prove Theorem 3, we consider the automorphism group of Cartesian products and their properties as determined by Sabidusi [23]. On the other hand, the study developed by Boutin [5] is based on characteristic matrices. We refer the reader to the work done by Boutin [5] for results on the determining number of Cartesian powers of prime connected graphs.

Proposition 4. [11] Let $P_{t}, P_{m}$ be two paths of order $t$ and $m$, respectively where $t, m \geqslant 2$. It holds that,

$$
\operatorname{Det}\left(P_{t} \square P_{m}\right)= \begin{cases}2 & \text { if } m=t=2 \text { or } 3 \\ 1 & \text { otherwise. }\end{cases}
$$

The following result provides the metric dimension of $P_{t} \square P_{m}$.

Proposition 5. Let $P_{t}, P_{m}$ be two paths with $t, m \geqslant 2$. Then $\beta\left(P_{t} \square P_{m}\right)=2$.

Proof. To prove that $\beta\left(P_{t} \square P_{m}\right)=2$ use the fact that $\beta(G) \leqslant \beta\left(G \square P_{m}\right) \leqslant \beta(G)+1$ (see $[6]), \beta\left(P_{m}\right)=1$, and so $1 \leqslant \beta\left(G \square P_{m}\right) \leqslant 2$. Obviously, two vertices are necessary and sufficient to obtain a metric basis of these graphs (see Figure 6).
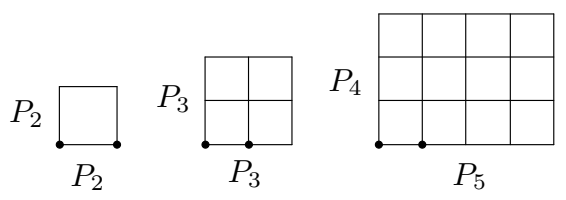

Figure 6: Metric basis of $P_{t} \square P_{m}$.

The connection between the determining number and the metric dimension, and Theorem 3 enable us to compute the determining number of the following Cartesian products. 
Proposition 6. Let $K_{t}, C_{t}$, and $P_{t}$ be the complete, cycle, and path graphs of order $t$, respectively.

(1) For every graph $H$ and integer $t \geqslant 2 \beta(H)+1$ it holds that $\operatorname{Det}\left(K_{t} \square H\right)=t-1$.

(2) $\operatorname{Det}\left(P_{t} \square C_{m}\right)=2$ whenever $t \geqslant 2$ and $m \geqslant 3$.

(3) For every $t \geqslant 2$ and $m \geqslant 2$ the following holds:

$$
\operatorname{Det}\left(K_{t} \square P_{m}\right)= \begin{cases}2 & \text { if } t=m=2, \\ 1 & \text { if } t=2 \text { and } m \neq 2, \\ t-1 & \text { if } t \geqslant 3 .\end{cases}
$$

(4) For every $t \geqslant 2$ and $m \geqslant 3$ the following holds:

$$
\operatorname{Det}\left(K_{t} \square C_{m}\right)= \begin{cases}2 & \text { if } t \leqslant 3 \text { and } m \neq 3, \\ 3 & \text { if } t=m=3, \\ t-1 & \text { if } t \geqslant 4 .\end{cases}
$$

(5) For every $t, m \geqslant 3$ we have

$$
\operatorname{Det}\left(C_{t} \square C_{m}\right)= \begin{cases}3 & \text { if } t=m=3 \text { or } t=m=4, \\ 2 & \text { otherwise. }\end{cases}
$$

Proof. We proceed by cases:

(1) By Lemma 5 and taking into account that $\beta\left(K_{t} \square H\right)=t-1$ if $t \geqslant 2 \beta(H)+1$ (see Theorem 5.3 in [6]) we have,

$$
t-1 \leqslant \max \left\{\operatorname{Det}\left(K_{t}\right), \operatorname{Det}(H)\right\} \leqslant \operatorname{Det}\left(K_{t} \square H\right) \leqslant \beta\left(K_{t} \square H\right)=t-1
$$

therefore $\operatorname{Det}\left(K_{t} \square H\right)=t-1$.

(2) It is a straightforward consequence of Theorem 3, using the fact that $\operatorname{Det}\left(P_{t}\right)=1$ and $\operatorname{Det}\left(C_{m}\right)=2$.

(3) Except for the case $t=m=2$, this comes directly from Theorem 3. Case $t=m=2$ comes from Proposition 4 .

(4) It is a consequence of Theorem 3, using the fact that $\operatorname{Det}\left(K_{t}\right)=t-1$ and $\operatorname{Det}\left(C_{m}\right)=2$. It only remains to prove the case $\operatorname{Det}\left(K_{3} \square C_{3}\right)$ which is shown in the next item 5 as $\operatorname{Det}\left(C_{3} \square C_{3}\right)$.

(5) Theorem 3 lets us conclude that $\operatorname{Det}\left(C_{t} \square C_{m}\right)=2$ if $t \neq m$. It remains to prove that $\operatorname{Det}\left(C_{m} \square C_{m}\right)=2$ whenever $m \geqslant 5$, and $\operatorname{Det}\left(C_{m} \square C_{m}\right)=3$ if $m=3$ or $m=4$. Note that Lemma 5 gives $\operatorname{Det}\left(C_{m} \square C_{m}\right) \geqslant 2$. We denote the vertex set of $C_{m}$, as $\{0,1, \ldots m-1\}$, so vertices in $C_{m} \square C_{m}$ are pairs $(i, j)$ with $0 \leqslant i, j \leqslant m-1$.

Claim 1. If a vertex $(i, j)$ in $C_{m} \square C_{m}, m \geqslant 5$, and two of its neighbors not both in the same row or column, are fixed by $f \in \operatorname{Aut}\left(C_{m} \square C_{m}\right)$, then all neighbors of $(i, j)$ are fixed by $f$. Suppose, without loss of generality, that $(i, j),(i, j-1),(i+1, j)$ are fixed by $f$ and that the other two neighbors of $(i, j)$ are not fixed, then $f(i, j+1)=(i-1, j)$ and $f(i-1, j)=(i, j+1)$ (see Figure 7$)$. But $(i, j-1)$ and $(i-1, j)$ have two common neighbors, however $f(i, j-1)$ and $f(i-1, j)$ have just one common neighbor (note that $m \geqslant 5)$. Hence $f$ must fix all neighbors of $(i, j)$. 


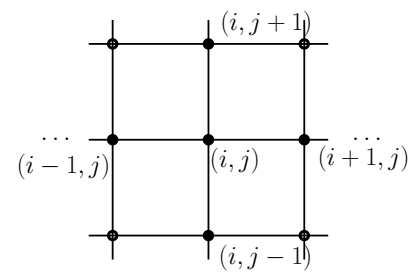

Figure 7: A graphical justification of Claim 1.

Similar arguments provides the following.

Claim 2. If a vertex $(i, j)$ in $C_{m} \square C_{m}, m \geqslant 5$, and all its neighbors are fixed by $f \in \operatorname{Aut}\left(C_{m} \square C_{m}\right)$, them $f=i d$.

Both claims let us conclude that a non identity automorphism can not fix simultaneously a vertex and two of its neighbors which are not located in the same row or column.

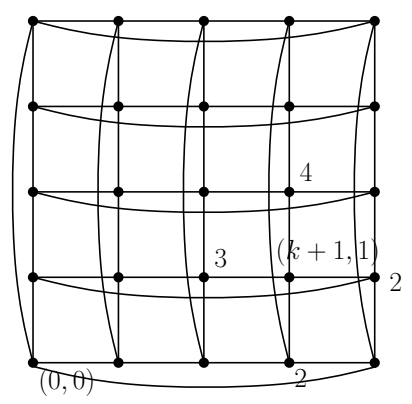

(a)

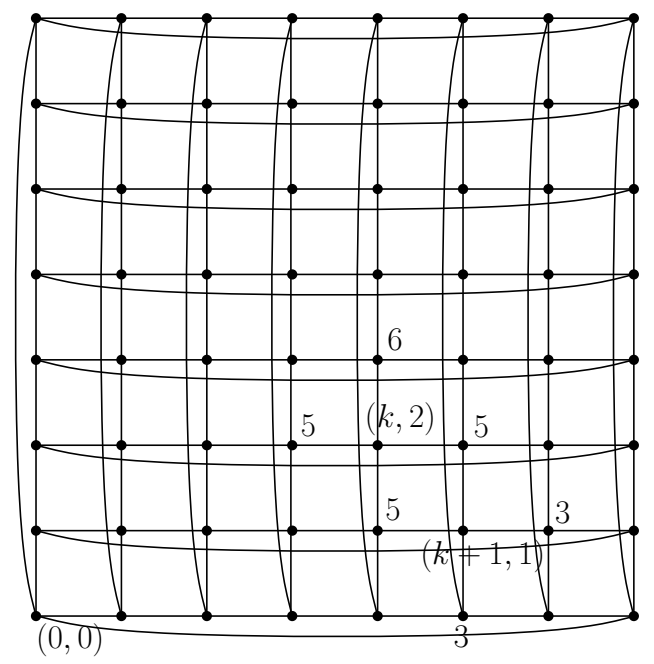

(b)

Figure 8: Any automorphism on $C_{m} \square C_{m}$ with $m \geqslant 5$ that fixes $(0,0)$ and $(k+1,1)$ must be the identity.

We show that $\operatorname{Det}\left(C_{m} \square C_{m}\right)=2$ whenever $m \geqslant 5$. We shall show that $\{(0,0),(k+$ $1,1)\}$, with $k=\left\lfloor\frac{m}{2}\right\rfloor$, is a determining set of $C_{m} \square C_{m}$. Suppose that $f \in \operatorname{Aut}\left(C_{m} \square C_{m}\right)$ fixes both $(0,0)$ and $(k+1,1)$. If $m=2 k+1, k \geqslant 2$, it is straightforward to prove that distances between $(0,0)$ and the neighbors of $(k+1,1)$ are the following: $d((0,0)(k+$ $1,0))=d((0,0),(k+2,1))=k, d((0,0),(k, 1))=k+1$ and $d((0,0),(k+1,2))=k+2$ (see Figure 8(a) where the labels of vertices are the distances to the point $(0,0))$ ). So $f$ must fix both $(k, 1)$ and $(k+1,2)$, and thus $f$ is the identity.

If $m=2 k, k \geqslant 2$, distances are $d((0,0)(k+1,0))=d((0,0),(k+2,1))=k-1$, 
$d((0,0),(k, 1))=d((0,0),(k+1,2))=k+1$. Suppose that $f$ is not the identity automorphism, then $f(k, 1)=(k+1,2)$ and $f(k+1,2)=(k, 1)$. Consider now the vertex $(k, 2)$, which is the second common neighbor of both $(k, 1)$ and $(k+1,2)$, the other one is $(k+1,1)$, fixed by $f$, so $(k, 2)$ is also fixed by $f$. Distances between $(0,0)$ and neighbors of $(k, 2)$ are $d((0,0),(k-1,2))=d((0,0),(k, 1))=d((0,0),(k+1,2))=k+1$ and $d((0,0),(k, 3))=k+3$ (see Figure $8(\mathrm{~b}))$, so $f$ must fix both $(k-1,2)$ and $(k, 3)$, which is not possible since $f$ is not the identity. This concludes the case $m=t \geqslant 5$.

It is easy to prove that, for every $u, v \in V\left(C_{4} \square C_{4}\right)$ there always exists a nontrivial automorphism $f \in \operatorname{Aut}\left(C_{4} \square C_{4}\right)$ so that $f(u)=u$, and $f(v)=v$. Analogously $\operatorname{Det}\left(C_{3} \square C_{3}\right)>2$.

On the other hand, the set of vertices $S=\{(0,0),(1,0),(0,1)\}$ is a determining set of both $C_{3} \square C_{3}$ and $C_{4} \square C_{4}$, since every column and every row of the Cartesian product is fixed by the action of any automorphism on $S$. Thus, we conclude that $\operatorname{Det}\left(C_{m} \square C_{m}\right)=3$ whenever $m=3$ or $m=4$.

Notice that Theorem 1 in [5] implies that $\operatorname{Det}\left(C_{m} \square C_{n}\right)=2$ whenever $m \neq n$. The above result includes the case $m=n$.

We next compute the determining number of $K_{t} \square K_{m}$. Observe that two vertices of this graph are adjacent if and only if they are located in a common row or column. Let $S \subseteq V\left(K_{t} \square K_{m}\right)$. A row or column is said to be empty if it contains no vertex in $S$. A vertex $v \in S$ is lonely (see [6]) if it is the only vertex of $S$ in its row and column. Figure 9 (a) shows instances of lonely and non-lonely vertices in $K_{7} \square K_{7}$.

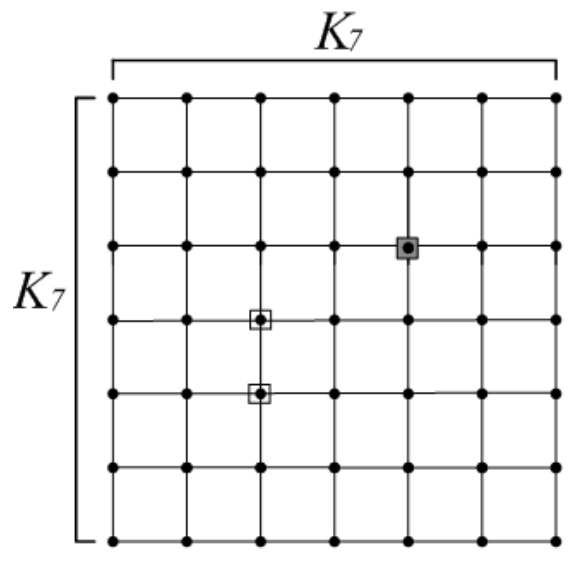

(a)

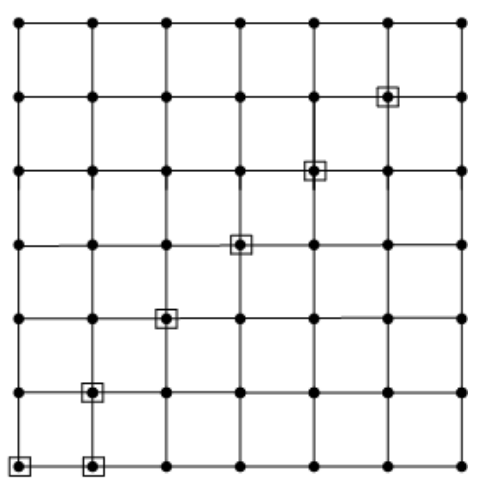

(b)

Figure 9: (a) The squared vertices form a set $S \subseteq V\left(K_{7} \square K_{7}\right)$ in which there are two non-lonely vertices and one lonely vertex (the darkened one), (b) a minimum determining set of $K_{7} \square K_{7}$. 
Lemma 6. For $t \geqslant 2$, a set $S \subseteq V\left(K_{t} \square K_{t}\right)$ is a determining set of $K_{t} \square K_{t}$ if and only if

(a) There is at most one empty row and at most one empty column, and

(b) There are at most $|S|-2$ lonely vertices.

Proof. $(\Longrightarrow)$ Assume that $S \subseteq V\left(K_{t} \square K_{t}\right)$ is a determining set of $K_{t} \square K_{t}$. By Lemma 5, the projection of $S$ onto $K_{t}$ is a determining set of $K_{t}$. Since $\operatorname{Det}\left(K_{t}\right)=t-1$ then condition $(a)$ holds.

Suppose now on the contrary that all the vertices of $S$ are lonely vertices, we can rename vertices in such a way that $S \subseteq\{(x, x) \mid 0 \leqslant x \leqslant t-1\}$ (note that both factors are complete graphs). Thus, there exists a non identity automorphism $f \in \operatorname{Aut}\left(K_{t} \square K_{t}\right)$ so that $f(i, j)=(j, i)$. Therefore, $S$ is not a determining set of $K_{t} \square K_{t}$ which leads to the desired contradiction.

$(\Longleftarrow)$ Assume now that $S \subseteq V\left(K_{t} \square K_{t}\right)$ is a set satisfying $(a)$ and $(b)$. Consider an automorphism $f \in \operatorname{Aut}\left(K_{t} \square K_{t}\right)$ such that $f(u)=u$ for all $u \in S$. Suppose on the contrary that $f$ is not the identity. Since there is at least one vertex of $S$ in every row except possibly one and in every column except possibly one, $f$ neither interchanges two rows nor two columns of $K_{t} \square K_{t}$. Thus, $f$ maps rows into columns and therefore it can fix at most one point in each row and in each column. However, condition $(b)$ implies that there exists one row (or column, or both) in which we have two vertices of $S$ fixed by $f$. The contradiction follows.

Proposition 7. For every $t, m \geqslant 2$ the following holds:

$$
\operatorname{Det}\left(K_{t} \square K_{m}\right)= \begin{cases}\max \{t-1, m-1\} & \text { if } t \neq m, \\ t & \text { if } t=m .\end{cases}
$$

Proof. The case $t \neq m$ is a straightforward consequence of Theorem 3, since $K_{t}$ and $K_{m}$ are relative prime graphs. Assume then that $t=m \geqslant 4$ (the case $K_{3} \square K_{3}$ was shown in item 5 of Proposition 6 as $\left.C_{3} \square C_{3}\right)$. To prove that $\operatorname{Det}\left(K_{t} \square K_{t}\right)=t$, it suffices to show that $t$ is the minimum number of vertices needed to satisfy conditions $(a)$ and $(b)$ of Lemma 6 . Clearly, if we consider $t-1$ vertices of $K_{t} \square K_{t}$ and at least two of them are non-lonely vertices then there are either two empty rows or two empty columns, what contradicts condition $(a)$. Moreover, the set $S=\{(x, x) \mid 0 \leqslant x \leqslant t-2\} \cup\{(1,0)\}$ satisfies items $(a)$ and $(b)$ of Lemma 6 (see Figure $9(\mathrm{~b})$ ). Thus, $\operatorname{Det}\left(K_{t} \square K_{t}\right)=t$.

Table 1 summarizes the results obtained in this section on the determining number, and the corresponding known-results on the metric dimension of Cartesian products (taken from $[6,21])$. Notice that the case $\operatorname{Det}\left(C_{3} \square C_{3}\right)=\operatorname{Det}\left(K_{3} \square K_{3}\right)=\operatorname{Det}\left(K_{3}^{2}\right)=3$ matches the formula $\operatorname{Det}\left(K_{3}^{k}\right)=\left\lceil\log _{3}(2 k+1)\right\rceil+1$ obtained by Boutin [5] for $k=2$.

Remark 2. The graph $K_{t} \square K_{m}$ is the first instance of Cartesian product of graphs in which the difference between the metric dimension and the determining number is arbitrarily large whenever $m \leqslant t \leqslant 2 m-1$. Indeed, $\beta\left(K_{t} \square K_{m}\right)-\operatorname{Det}\left(K_{t} \square K_{m}\right)$ is $\Omega\left(\frac{2 m-t}{3}\right)$ where $\left|V\left(K_{t} \square K_{m}\right)\right|=n=t \cdot m$. In particular, for $m=t, \beta\left(K_{t} \square K_{t}\right)-\operatorname{Det}\left(K_{t} \square K_{t}\right)$ is $\Omega\left(\frac{\sqrt{n}}{3}\right)$. 
Table 1: Summary of results.

\begin{tabular}{|c|c|c|}
\hline$G$ & $\operatorname{Det}(G)$ & $\beta(G)$ \\
\hline$P_{t} \square P_{m}$ & $\begin{array}{ll}2 & \text { if } t=m=2,3 \\
1 & \text { otherwise }\end{array}$ & 2 \\
\hline$P_{t} \square C_{m}$ & 2 & 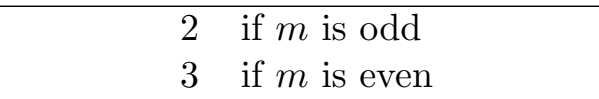 \\
\hline$C_{t} \square C_{m}$ & $\begin{array}{ll}3 & \text { if } t=m=3,4 \\
2 & \text { otherwise }\end{array}$ & $\begin{array}{ll}3 & \text { if } t \text { or } m \text { are odd } \\
4 & \text { if } t \text { and } m \text { are even }\end{array}$ \\
\hline$K_{t} \square P_{m}$ & $t-1$ & $t-1$ \\
\hline$K_{t} \square C_{m}$ & $\begin{array}{ll}2 & \text { if } t=3 \text { and } m \neq 3 \\
3 & \text { if } t=m=3 \\
t-1 & \text { if } t \geq 4\end{array}$ & $\begin{array}{ll}3 & \text { if } t=4 \text { and } m \text { is even } \\
4 & \text { if } t=4 \text { and } m \text { is odd } \\
t-1 & t \geq 5\end{array}$ \\
\hline $\begin{aligned} & K_{t} \square H \\
& t \geq 2 \beta(H)+1\end{aligned}$ & $t-1$ & $t-1$ \\
\hline$K_{t} \square K_{m}$ & $\begin{array}{ll}\max \{t-1, m-1\} & \text { if } t \neq m \\
t & \text { if } t=m\end{array}$ & $\begin{array}{ll}\left\lfloor\frac{2}{3}(t+m-1)\right\rfloor & \text { if } m \leq t \leq 2 m-1 \\
t-1 & \text { if } t \geq 2 m-1\end{array}$ \\
\hline
\end{tabular}

Table 1 shows that in the rest of cases both parameters are equal or the difference is at most 2 .

To conclude this section, consider the hypercube $Q_{n}$ which is the graph whose vertices are the $n$-dimensional binary vectors, where two vertices are adjacent if they differ in exactly one coordinate. It is well-known that,

$$
Q_{n}=\underbrace{K_{2} \square K_{2} \square \cdots \square K_{2}}_{n} .
$$

The determining number of the hypercube is given by $\operatorname{Det}\left(Q_{n}\right)=\left\lceil\log _{2} n\right\rceil+1$ (see [5]). On the other hand, the works done by Erdős and Rényi [10], and Lindström [20] lead to

$$
\lim _{n \rightarrow \infty} \beta\left(Q_{n}\right) \cdot \frac{\log n}{n}=2
$$

Therefore, $\beta\left(Q_{n}\right)-\operatorname{Det}\left(Q_{n}\right)$ is asymptotically $\Omega\left(\frac{2 n-\log ^{2} n}{\log n}\right)$.

\section{$5 \quad$ Lower bounds on $\beta(G)-\operatorname{Det}(G)$}

The studies developed in the two previous sections let us answer the question asked by Boutin [4]: Can the difference between the determining number and the metric dimension of a graph of order $n$ be arbitrarily large? In Sections 3 and 4 we have shown that $\beta(T)-\operatorname{Det}(T)=\Omega(\sqrt{n})$ for some trees, and $\beta\left(K_{t} \square K_{t}\right)-\operatorname{Det}\left(K_{t} \square K_{t}\right)=\Omega\left(\frac{\sqrt{n}}{3}\right)$. The following result improves these lower bounds. 
Proposition 8. There exists a 2-connected graph $G$ of order $n$ such that

$$
\beta(G)-\operatorname{Det}(G)=\Omega\left(\frac{2 n}{5}\right) .
$$

Proof. For $n \geqslant 5$, let $G=W_{1, n-1}$ be the wheel formed by joining a single vertex $v$ to all vertices of an $(n-1)$-cycle, denoted by $C_{n-1}$. Every automorphism of $W_{1, n-1}$ has to fix $v$, since its degree is at least 4 and the rest of the vertices have degree 3 . Thus, it suffices to consider the action of $f \in \operatorname{Aut}\left(W_{1, n-1}\right)$ on a minimum determining set of $C_{n-1}$ to determine the action of $f$ on $W_{1, n-1}$. Hence, a set of two non-antipodal vertices of $C_{n-1}$ is a minimum determining set of $W_{1, n-1}$. Thus, the determining number of the wheel graphs is always 2 independently of the number of vertices.

On the other hand, Shanmukha and Sooryanarayana [24] show that $\beta\left(W_{1, n-1}\right)$ increases with the number of vertices:

$$
\forall k \in \mathbb{N}, \quad \beta\left(W_{1, x+5 k}\right)= \begin{cases}3+2 k & \text { if } \quad x=7 \text { or } 8 \\ 4+2 k & \text { if } \quad x=9,10 \text { or } 11 .\end{cases}
$$

Therefore the difference between the two parameters is:

$$
\beta\left(W_{1, x+5 k}\right)-\operatorname{Det}\left(W_{1, x+5 k}\right)= \begin{cases}2 k+1=\Omega\left(\frac{2 n}{5}\right) & \text { if } \quad x=7 \text { or } 8, \\ 2 k+2=\Omega\left(\frac{2 n}{5}\right) & \text { if } \quad x=9,10 \text { or } 11 .\end{cases}
$$

where $n=\left|V\left(W_{1, x+5 k}\right)\right|=x+5 k+1$.

We want to stress that we have also computed the metric dimension and the determining number of graphs formed by joining wheels in different ways using the explosion technique, that is, by joining the central vertex of a wheel $W_{1, m}$ to any vertex of $G$ as in [24]. As the graph $G$, it has been used $P_{n}, C_{n}$ and $K_{n}$. We have also considered the case of adding edges to the wheel graph $W_{1, n-1}$ in different ways trying to increase the metric dimension, and maintaining the determining number to be a constant. Nevertheless, all these families of graphs give rise to at most the same $\Omega\left(\frac{2 n}{5}\right)$ lower bound. We have not been able to improve this lower bound. Thus, there is a gap between $\frac{2 n}{5}$ and $n-2$.

\section{References}

[1] M. O. Albertson and D. L. Boutin. Using determining sets to distinguish Kneser graphs. Electron. J. Combin., 14(1): Research paper 20 (Electronic), 2007.

[2] M. O. Albertson and K. Collins. Symmetry breaking in graphs. Electron. J. Combin., 3 (1996) R 18.

[3] L. W. Beineke and R. J. Wilson. Graph connections: Relationships between graph theory and other areas of mathematics. Volume 5 of Oxford Lecture Series in Mathematics and its applications. The Clarendon Press Oxford University Press, New York, 1997. 
[4] D. L. Boutin. Identifying graphs automorphisms using determining sets. Electron. J. Combin., 13(1): Research paper 78 (Electronic), 2006.

[5] D. L. Boutin. The determining number of a Cartesian product. Journal of Graph Theory, 61(2), pp. 77-87, 2009.

[6] J. Cáceres, C. Hernando, M. Mora, I. M. Pelayo, M. L. Puertas, C. Seara, and D. R. Wood. On the metric dimension of Cartesian products of graphs. SIAM Journal on Discr. Math., 21(2), pp. 423-441, 2007.

[7] K. M. Chao and B. Y. Wu. A note on excentricities, diameters and radii. An excerpt from the book "Spanning trees and optimization problems", Chapman and Hall/CRC Press, USA, 2004.

[8] G. Chartrand, L. Eroh, M. A. Johnson, and O. R. Oellermann. Resolvability in graphs and the metric dimension of a graph. Discrete Appl. Math., 105(1-3), pp. 99-113, 2000.

[9] K. L. Collins and J. D. Laison. Fixing numbers of Kneser graphs. Manuscript.

[10] P. Erdős and A. Rényi. On two problems of information theory. Magyar Tud. Akad. Mat. Kutató Int. Közl., 8, pp. 229-243, 1963.

[11] D. Erwin and F. Harary. Destroying automorphisms by fixing nodes. Discrete Math., 306, pp. 3244-3252, 2006.

[12] C. R. Gibbons and J. D. Laison. Fixing numbers of graphs and groups. The Electronic Journal of Combinatorics, 16: Research paper 39 (Electronic), 2009.

[13] F. Harary. Methods of destroying the symmetries of a graph. Bull. Malasyan Math. Sc. Soc., 24(2), pp. 183-191, 2001.

[14] F. Harary and R. A. Melter. On the metric dimension of a graph. Ars Combinatoria, 2, pp. 191-195, 1976.

[15] C. Hernando, M. Mora, I. M. Pelayo, C. Seara, and D. R. Wood. Extremal graph theory for metric dimension and diameter. Electronic Notes in Discr. Math., 29, pp. 339-343, 2007.

[16] J. E. Hopcroft and R. E. Tarjan. Isomorphism of planar graphs. In: R.E. Miller, J.W. Thatcher (eds.) Complexity of Computer Computations, Plenum Press, 1972, pp. 131-150.

[17] W. Imrich and S. Klavzar. Product graphs: Structure and Recognition. Wiley-Interscience, 2000.

[18] C. Jordan. Sur les assemblages de lignes. J. Reine Angew. Math, 70, pp. 185-190, 1869.

[19] S. Khuller, B. Raghavachari, and A. Rosenfeld. Landmarks in graphs. Discrete Appl. Math., 70(3), pp. 217-229, 1996.

[20] B. Lindström. On a combinatory detection problem. I. Magyar Tud. Akad. Mat. Kutató Int. Közl., 9, pp. 195-207, 1964. 
[21] J. Peters-Fransen and O. R. Oellermann. The metric dimension of Cartesian products of graphs. Util. Math., 69, pp 33-41, 2006.

[22] C. Poisson and P. Zhang. The metric dimension of unicycle graphs. J. Combin. Math. Combin. Comput. 40, pp. 17-32, 2002.

[23] G. Sabidussi. Graph multiplication. Math. Zeitschr., 72, pp. 446-457, 1960.

[24] B. Shanmukha and B. Sooryanarayana. Metric dimension of wheels. Far East J. Appl. Math., 8(3), pp. 217-229, 2002.

[25] P. J. Slater. Leaves of trees. In: Proc. 6th Southeastern Conf. on Combinatorics, Graph Theory and Computing, 14, pp 549-559, 1975.

[26] D. B. West. Introduction to Graph Theory. Prentice Hall, 1996. 